\title{
Inhibition as a Factor in the Maintenance of the Diversity of Microbial Ecosystems
}

\author{
By MAX J. DE FREITAS AND A. G. FREDRICKSON \\ Department of Chemical Engineering and Materials Science, University of Minnesota, \\ Minneapolis, Minnesota 55455, U.S.A.
}

(Received 31 January 1978)

\begin{abstract}
Simple models of growth, nutrient utilization, product formation and inhibitor action are used to show that two populations competing for a single nutrient which is limiting their rates of growth can coexist in an ideal chemostat if at least one of the populations produces an autoinhibitor. Production of a substance that is not autoinhibitory but inhibits instead the competitor population (antagonism) will not allow coexistence but can give a population a chance to exclude its competitor under conditions where it would otherwise be excluded by the competitor. These results show that production of autoinhibitors can play an important role in maintaining the species diversity of microbial ecosystems.
\end{abstract}

\section{INTRODUCTION}

Definitions. Any substance in the environment is said to be rate limiting for a population if, during the course of the growth situation being considered, changes in the concentration of the substance are sufficient to cause measurable changes in the growth rate of the population. Thus the concentrations of all substances that are rate limiting for some population must appear in the mathematical description of a microbial ecosystem.

Any substance that is consumed by a population during its growth is called a nutrient. It is assumed that no nutrient is present at a concentration which, if increased, would cause a decrease in the growth rate of any population; such inhibition of growth by a rate limiting nutrient leads to mathematical complications (Aris \& Humphrey, 1977) that we prefer to avoid.

Two populations in a common environment are said to compete with one another for nutrient or nutrients if there are rate limiting substances for both populations among the set of nutrients consumed by both populations. A less general form of competition is considered in the models discussed below; these assume that competition involves only a single rate limiting nutrient.

An ideal chemostat is one in which the culture within it is spatially homogeneous and for which the ratio of wetted surface area to culture volume is exceedingly small so that wall effects can be ignored. The external circumstances of a chemostat are the temperature, pressure, illumination, etc., in its immediate surroundings.

A primary substance in the medium is one that is present therein because it enters with the inputs to the chemostat. A secondary substance is one that is produced and excreted by the populations growing in the chemostat. We assume that there are no rate limiting substances that are both primary and secondary substances.

In laboratory experiments on competition of two microbial populations in a chemostat for a single rate limiting nutrient, the density of one of the competitors has always declined toward undetectable levels (Harder \& Veldkamp, 1971; Jannasch, 1967, 1968; Jost et al., 
1973; Meers, 1971; Meers \& Tempest, 1968; Megee et al., 1972; Tempest, Dicks \& Meers, 1967). Indeed the mathematical analysis of competition in a chemostat by Powell (1958) predicted these experimental results. Thus, both experiment and theory assert that competition for a single rate limiting nutrient leads to exclusion of one of the competitors. The foregoing principle has been stated in a more far-reaching manner by Hardin (1960) in his competitive exclusion principle.

Nevertheless, non-laboratory ecosystems often exhibit coexistence of many populations that have overlapping nutrient requirements. This is Hutchinson's (1961) paradox of the plankton. Ecologists often propose to resolve the paradox by pointing out that ecosystems are not spatially homogeneous and that external circumstances and inputs to the systems are not time invariant as they would be in a chemostat. Certainly, spatial heterogeneity can allow competitors to coexist in an ecosystem, and variation of external circumstances or inputs to the system may also allow competitors to coexist if they have some appropriate physiological peculiarities (van Gemerden, 1974; Williams, 1971). However, it is by no means clear, for example, that periodic variation of the flow rate to a chemostat will lead to coexistence of two bacterial populations that compete for the same rate limiting nutrient.

Another circumstance that might allow competitors to coexist is the presence of a common predator, as in the three-population experiment reported by Jost et al. (1973). This, however, cannot of itself resolve the paradox of the plankton because the problem of what allows the top predators (if there is, indeed, more than one) to coexist will remain.

Can competitors, not subject to predation (or parasitism), coexist in a system that is spatially homogeneous and has time invariant but otherwise unconstrained external circumstances and inputs? It can be shown that a necessary, but not sufficient, condition for such coexistence is that the number of substances present in the abiotic environment that have limiting effects on the net growth rates of competitors must equal or exceed the number of competing populations present. This is not a sufficient condition for coexistence because it does not guarantee that steady states of coexistence will be stable ones.

Recently, Taylor \& Williams (1975) and Schuelke (1976) have shown that mathematical models of competition in an ideal chemostat have stable coexistence steady states if the number of primary nutrients that have rate limiting effects on the competitors equals or exceeds the number of competing populations. Specifically, Taylor \& Williams showed that their model of competition of two populations for two common rate limiting primary nutrients has a stable coexistence steady state. Schuelke considered a model for competition of two populations for a common rate limiting primary nutrient, but with each population also limited by a second, non-common, primary nutrient. This model yielded a stable coexistence steady state. The coexistence steady states in these two studies were predicted to be stable over ranges of values of chemostat dilution rate and concentrations of rate limiting nutrients in the feed. This contrasts with the situation considered by Powell (1958) in which coexistence in a chemostat of two populations which compete for a single rate limiting primary nutrient is possible only at one fixed value of the dilution rate.

Diversity of primary nutrients may thus be a factor in the maintenance of diversity of species in ecosystems. In many situations, however, the number of such nutrients may be too small to account for the observed species diversity, and one looks for other circumstances that will allow species diversity to be maintained.

It is not necessary that rate limiting substances be present in a system only because they are introduced into it from its surroundings. The activities of the populations present convert primary substances into new or secondary substances, and these may also exert rate limiting effects in the system. In this way, a small number of primary substances may be multiplied into a larger number of total (primary plus secondary) substances, and the number of populations potentially able to coexist in the system will be increased.

Experimental and conceptual examples of the foregoing process are found, for example, in the papers of Megee et al. (1972), Lee, Fredrickson \& Tsuchiya (1976) and Meyer, 
Tsuchiya \& Fredrickson (1975). These examples of maintenance of species diversity through the production of secondary rate limiting substances all involve situations in which the secondary substances stimulate the populations whose growth rates they affect. The question therefore arises, can production of secondary substances which inhibit the growth rates of populations play a role in maintaining the diversity of species in ecosystems? This is the question to be explored in the present paper. Our methodology has been to construct and analyse simple models for the growth of two populations in an ideal chemostat when various patterns of inhibitor production and inhibitor effects are postulated, and when the populations compete for a primary nutrient whose availability has a rate limiting effect on each of them. In each case, the object will be to see if stable coexistence steady states are predicted by the model for ranges of values of the operating conditions (chemostat dilution rate and concentration of the rate limiting nutrient in the feed). Model equations will be written for chemostat situations only, not because such situations are important in nature, but rather because propagation in a chemostat is a definite and experimentally realizable situation (Williams, 1971).

\section{Case I. The competitors produce a common autoinhibitor}

We assume here that production of the autoinhibitor by each population is strictly growth-associated and that its effect on the growth rate of each population follows the modification of Monod's model used by Aiba \& Shoda (1969) and Wilkinson, Topiwala \& Hamer (1974). In experiments on autoinhibition of yeast by alcohol, Aiba \& Shoda (1969) found that the rate of alcohol production was not proportional to growth rate, but we shall ignore this complication here. Use of the Aiba \& Shoda expression for the rate of inhibitor formation would complicate analysis of the models but would hardly change the qualitative conclusions. We assume, in addition, that neither population consumes the inhibitor, that maintenance consumption of the rate limiting nutrient is negligible, that there is no wall growth etc. The model equations for this situation are then

$$
\begin{aligned}
& \frac{\mathrm{d} x_{\mathrm{j}}}{\mathrm{d} t}=-D x_{\mathrm{j}}+\mu_{\mathrm{j}} x_{\mathrm{j}}, \quad \mathrm{j}=1,2 \\
& \frac{\mathrm{d} i}{\mathrm{~d} t}=-D i+\sum_{\mathrm{j}=1}^{2} \alpha_{\mathrm{j}} \mu_{\mathrm{j}} x_{\mathrm{j}} \\
& \frac{\mathrm{d} s}{\mathrm{~d} t}=D\left(s_{\mathrm{f}}-s\right)-\sum_{\mathrm{j}=1}^{2} \frac{1}{Y_{\mathrm{j}}} \mu_{\mathrm{j}} x_{\mathrm{j}}
\end{aligned}
$$

where the specific growth rates are given by

$$
\mu_{\mathrm{j}}=\frac{\mu_{\mathrm{mj}} s}{\left(K_{\mathrm{j}}+s\right)\left[1+i / K_{\mathrm{j}}^{\prime}\right]}
$$

and where $x_{\mathrm{j}}, i$ and $s$ are the concentrations in the culture of, respectively, biomass of the jth population, inhibitor and rate limiting nutrient, $s_{\mathrm{P}}$ is the concentration in the feed of the rate limiting nutrient, $D$ is the dilution rate, and $\mu_{\mathrm{mj}}, K_{\mathrm{j}}, K_{\mathrm{j}}^{\prime}, \alpha_{\mathrm{j}}$ and $Y_{\mathrm{j}}$ are the model parameters for the $\mathrm{jth}$ population. The parameter $K_{\mathrm{j}}^{\prime}$ measures the effect of the inhibitor on the jth population; it is the inhibitor concentration at which the specific growth rate is reduced to half the value it would have at the same nutrient concentration if no inhibitor were present. Thus, small values of $K_{\mathrm{j}}^{\prime}$ indicate that the jth population is subject to considerable inhibition whereas large values of $K_{j}^{\prime}$ indicate the converse. The product $\alpha_{\mathfrak{j}} Y_{\mathrm{j}}$ is the amount of inhibitor formed when unit mass of the rate limiting nutrient is consumed by the jth population; this product will be called the yield of inhibitor by the jth population.

Clearly, the nature of solutions of equations (1) will depend on the model parameters used and on the operating conditions, $D$ and $s_{\mathrm{f}}$, imposed. This dependence can be summarized on an operating diagram (Jost et al., 1973). An operating diagram is a graph 


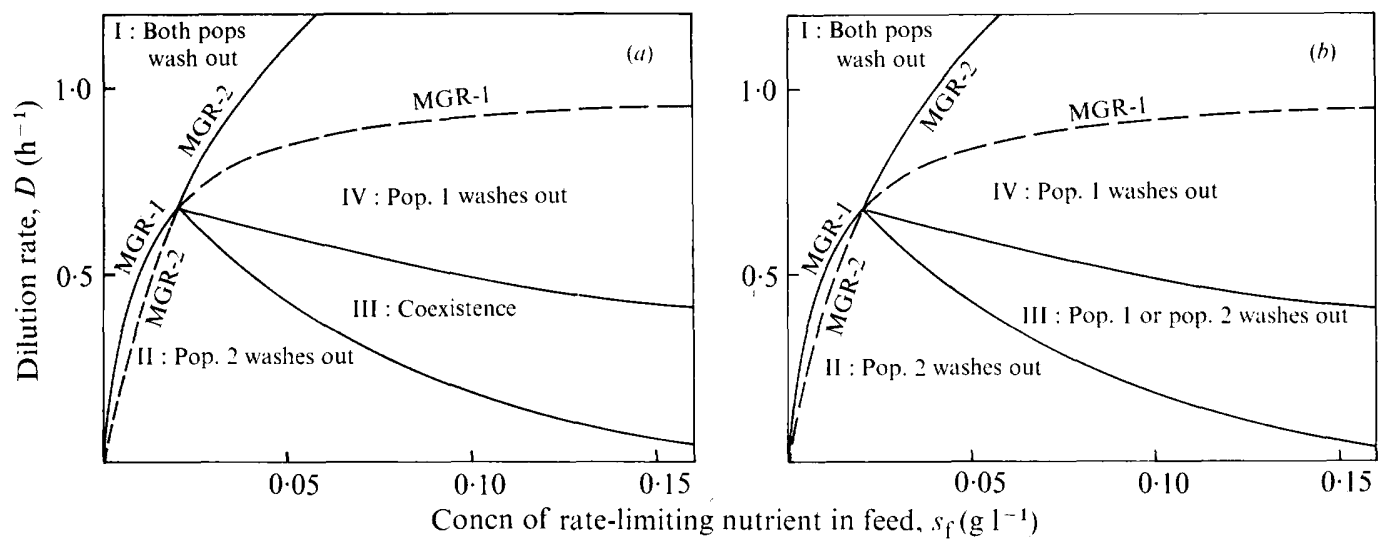

Fig. 1. Operating diagrams for Case I: the competitors produce a common autoinhibitor. The values of the model parameters used in constructing these diagrams are as follows:

$\begin{array}{ccccccccc} & \begin{array}{c}\mu_{\mathrm{m} 1} \\ \left(\mathrm{~h}^{-1}\right)\end{array} & \begin{array}{c}\mu_{\mathrm{m} 2} \\ \left(\mathrm{~h}^{-1}\right)\end{array} & \begin{array}{c}K_{1} \\ \left(\mathrm{~g} \mathrm{l}^{-1}\right)\end{array} & \begin{array}{c}K_{2} \\ \left(\mathrm{~g} \mathrm{l}^{-1}\right)\end{array} & \begin{array}{c}K_{1}^{\prime} \\ \left(\mathrm{g} \mathrm{l}^{-1}\right)\end{array} & \begin{array}{c}K_{2}^{\prime} \\ \left(\mathrm{g} \mathrm{l}^{-1}\right)\end{array} & \alpha_{1} Y_{1} & \alpha_{2} Y_{2} \\ (a) & 1.0 & 2 \cdot 0 & 0.01 & 0.04 & 0.01 & 0.006 & 4 \cdot 0 & 0.1 \\ (b) & 1.0 & 2.0 & 0.01 & 0.04 & 0.01 & 0.006 & 0.1 & 4.0\end{array}$

showing regions in the first quadrant of the $D-s_{f}$ plane where various steady state solutions of the model equations exist and are stable with respect to small perturbations. The model parameters are fixed for any single operating diagram that is prepared, but one can illustrate the effects of changes of model parameters on the nature of solutions of the model equations by preparing a separate operating diagram for each set of model parameters of interest. Preparation of operating diagrams is described in the Appendix.

Figure 1 shows operating diagrams constructed from equations (1). The difference between Fig. $1(a)$ and $1(b)$ is that the yields of inhibitor by the two populations are different. In Fig. 1, and in all other operating diagrams, the solid curves indicate loci of operating conditions at which a change in the nature of solutions of the model equations occurs; they are therefore called boundary curves. The dashed curves are extensions of boundary curves, but no change in the nature of solutions of the model equations occurs across them; they are included because they help one to understand the construction of the operating diagrams.

The equation $D=\mu_{\mathrm{mj}} \mathrm{s}_{\mathrm{f}} /\left(K_{\mathrm{j}}+s_{\mathrm{f}}\right)$ defines the maximum dilution rate at which a pure culture of the jth population can maintain itself in a chemostat when the concentration of rate limiting nutrient in the feed is $s_{\mathrm{p}}$. If $D$ is just slightly less than the value given by this equation, the pure culture of the jth population will persist, but its biomass concentration will be very small and the concentration of the autoinhibitor will be very small, so that the specific growth rate will be determined only by the availability of the nutrient. Hence, the curve of the foregoing equation on an operating diagram is labelled MGR-j, for Maximum Growth Rate of the jth population.

In Fig. 1(a), the first quadrant of the $D-S_{\mathrm{f}}$ (operating) plane is divided into four regions by boundary curves. In the portion of the operating plane above both MGR curves (region I), the only stable steady state which is real (i.e. has all concentrations non-negative) is that where both populations are washed out of the chemostat. In the other three regions, however, there exist stable steady states in which at least one population is present. In region II, population 2 is excluded from the chemostat but population 1 establishes itself. Conversely, in region IV, population 1 is excluded from the chemostat but population 2 establishes itself. In region III the only stable steady state is that in which the competing populations coexist with one another. Coexistence is stable over a finite range of values of the dilution rate, so it would not be necessary to hold this quantity exactly at some critical value in order 


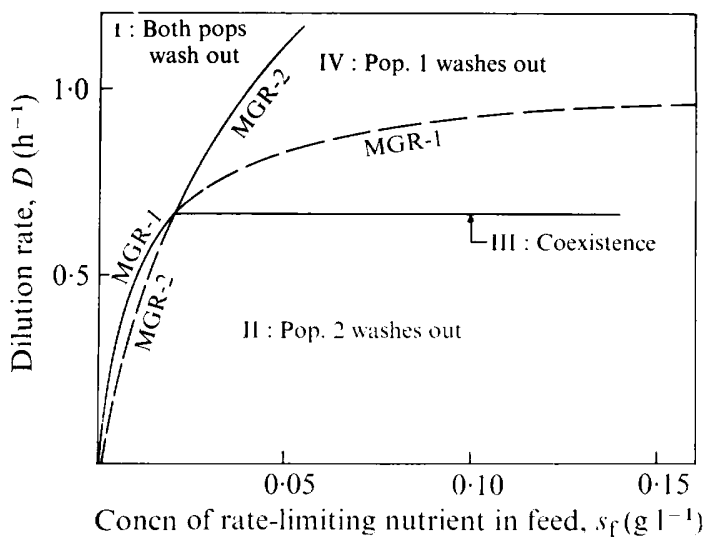

Fig. 2. Operating diagram for the case where the competitors produce no inhibitors. The values of $\mu_{\mathrm{m} 1}, \mu_{\mathrm{m} 2}, K_{1}$ and $K_{2}$ used in constructing this diagram are the same as those in Fig. 1, but $K_{1}^{\prime}$ and $K_{2}^{\prime}$ are both infinite (values of $\alpha$ and $Y$ are irrelevant to the construction of the diagram). If the ordinate of the MGR-2 curve exceeded that of the MGR-1 curve for all values of $s_{i}$, population 2 would exclude population 1 for all operating conditions lying under the MGR-2 curve.

to prevent a drift toward exclusion of one of the competitors. Thus, if model parameters and operating conditions are appropriate, the production of a common autoinhibitor can allow two competing populations to coexist with one another.

The model parameters used to construct Fig. 1(b) are exactly the same as those used to construct Fig. 1(a) except that the values of the inhibitor yields have been inverted. The boundary curves in Fig. $1(b)$ are exactly the same as those in Fig. $1(a)$ and the steady states that are stable in regions I, II and IV are the same. However, there is no region in Fig. 1(b) in which the coexistence steady state is stable. Instead, if operating conditions lie in region III of Fig. 1 $(b)$, both steady states where one population is washed out of the chemostat are stable with respect to small perturbations. Under such conditions, which population is excluded would depend on the initial conditions imposed. Coexistence is not possible at any set of operating conditions if the model parameters are those used to construct Fig. 1(b).

It is of interest to compare the operating diagrams of Fig. 1 with that in Fig. 2 for the case where neither population is affected by the inhibitor, i.e. the situation considered by Powell (1958). Regions I, II and IV exist and yield the same steady states as in Figs $1(a)$ and $1(b)$, but region III is reduced to the horizontal line $D=0.667 \mathrm{~h}^{-1}, s_{\mathrm{f}}>0.02 \mathrm{~g} \mathrm{l}^{-1}$. Along this line, coexistence is a neutrally stable steady state, but the practical impossibility of maintaining the dilution rate at the critical value $0.667 \mathrm{~h}^{-1}$ would result in exclusion of one or other of the populations if this situation were studied experimentally. The effect of the common autoinhibitor on the dynamics of competition is to expand the neutrally stable coexistence line of Fig. 2 into the stable domain (region III) of Figs $1(a)$ and $1(b)$ where, even if coexistence is not possible, either population has a chance of winning the 'struggle for existence'.

As one would expect, the operating diagrams shown in Fig. 1 would be qualitatively as well as quantitatively changed if the model parameters were changed sufficiently. Two effects of parameter changes are of interest. First, in Figs 1 and 2, the MGR curves for the two populations cross, and this is a necessary condition for the coexistence steady state to be even neutrally stable in the case (Fig. 2) where neither population is subject to autoinhibition. For if, in Fig. 2, the MGR-2 curve were below the MGR-1 curve at all values of $s_{\mathrm{f}}$, then washout of population 2 and establishment of population 1 would be the stable steady state for all operating conditions lying below the MGR-1 curve. Crossing of the MGR curves is also a necessary condition for coexistence in Case I, though it is not necessary in any of the cases which follow. 
Second, changed model parameters can also lead to situations in which two steady states coexistence of the competitors and exclusion of one of them - are stable with respect to small perturbations in certain domains of the operating plane. The result of competition in such a situation is not determined solely by the differential equations and parameters of the model, but also depends on the initial conditions imposed on the values of $x_{1}, x_{2}, i$ and $s$. Operating diagrams for situations of this kind are considerably more complex than those shown in Fig. 1, and no examples will be given here.

\section{Case II. Only one population produces and is affected by an autoinhibitor}

If it is assumed that one of the populations neither produces the inhibitor nor is affected by it, then the only interaction between the populations is competition for the common rate limiting nutrient. The effects of autoinhibition on the other population can nevertheless allow the competitors to coexist in a stable steady state over a range of operating conditions, as is shown below.

The equations of Case II are the same as those of Case I, but here we have to let $\alpha_{2}$ approach zero and $K_{2}^{\prime}$ approach infinity. This case therefore has fewer model parameters than does Case $I$, and consequently, one would expect there to be fewer qualitatively different operating diagrams. There are, in fact, only four such diagrams (Fig. 3).

In Fig. 3(a) the MGR curve of the population not subject to inhibition (population 2) lies above the MGR curve of the population subject to inhibition (population 1) for all values of $s_{\mathrm{f}}$. Coexistence is not possible at any set of operating conditions, and the population not subject to inhibition will exclude the population subject to inhibition at all operating conditions that lie below the upper MGR curve.

In Figs $3(b)$ and $3(c)$ the MGR curves of the two populations cross. Were it not for the autoinhibition of population 1 , the operating diagrams would become qualitatively the same as that shown in Fig. 2, with large dilution rates causing the exclusion of population 1 in the case of the parameters used for Fig. $3(b)$, but of population 2 in the case of the parameters used for Fig. 3(c). Autoinhibition of population 1 changes these pictures greatly (Figs $3 b$ and $3 c$ ). In particular, coexistence will be a stable steady state for dilution rates less than $0.667 \mathrm{~h}^{-1}$ in the case of Fig. 3(b), but greater than $0.667 \mathrm{~h}^{-1}$ in the case of Fig. 3(c), if the concentration of the rate limiting nutrient in the feed is appropriately greater than $0.02 \mathrm{~g} \mathrm{l}^{-1}$. Again, the effect of autoinhibition is to expand the coexistence line of Fig. 2 into the regions (III) shown in Figs $3(b)$ and $3(c)$. Thus, when one population is subject to autoinhibition, it would be unnecessary to maintain the dilution rate exactly at a critical value in order to obtain coexistence of the competitors.

Figure $3(d)$ shows the case where the MGR curve of the population subject to inhibition lies above the MGR curve of the population not subject to inhibition for all values of $s_{\mathrm{p}}$. Coexistence will be a stable steady state over a large range of operating conditions. In fact, since the boundary curve marked NFC (Nutrient Feed Condition, see Appendix for explanation) is asymptotic to MGR-2 at large values of $s_{\mathrm{f}}$ and has an intercept of

$$
K_{1}^{\prime}\left(K_{2} \mu_{\mathrm{m}_{1}}-K_{1} \mu_{\mathrm{m}_{2}}\right) / \alpha_{1} Y_{1} K_{1} \mu_{\mathrm{m}_{2}}
$$

on the $s_{\mathrm{f}}$ axis, it follows that coexistence will be a stable steady state at virtually all operating conditions at which the population not subject to autoinhibition can maintain itself in pure culture, provided that the other population is sufficiently affected by autoinhibition (i.e. provided that $K_{1}^{\prime}$ is small enough). In other words, curve NFC becomes identical with curve MGR-2 as $K_{1}^{\prime}$ decreases indefinitely.

Comparison of Figs 2 and 3 shows that autoinhibition allows the competitors to coexist only under conditions where the population subject to inhibition would exclude the population not subject to inhibition were it not for the action of the inhibitor. This necessary condition for coexistence points out the essential role of the inhibitor in allowing coexistence: it acts to make the growth rate of the population which is subject to inhibition partially self-limited. 

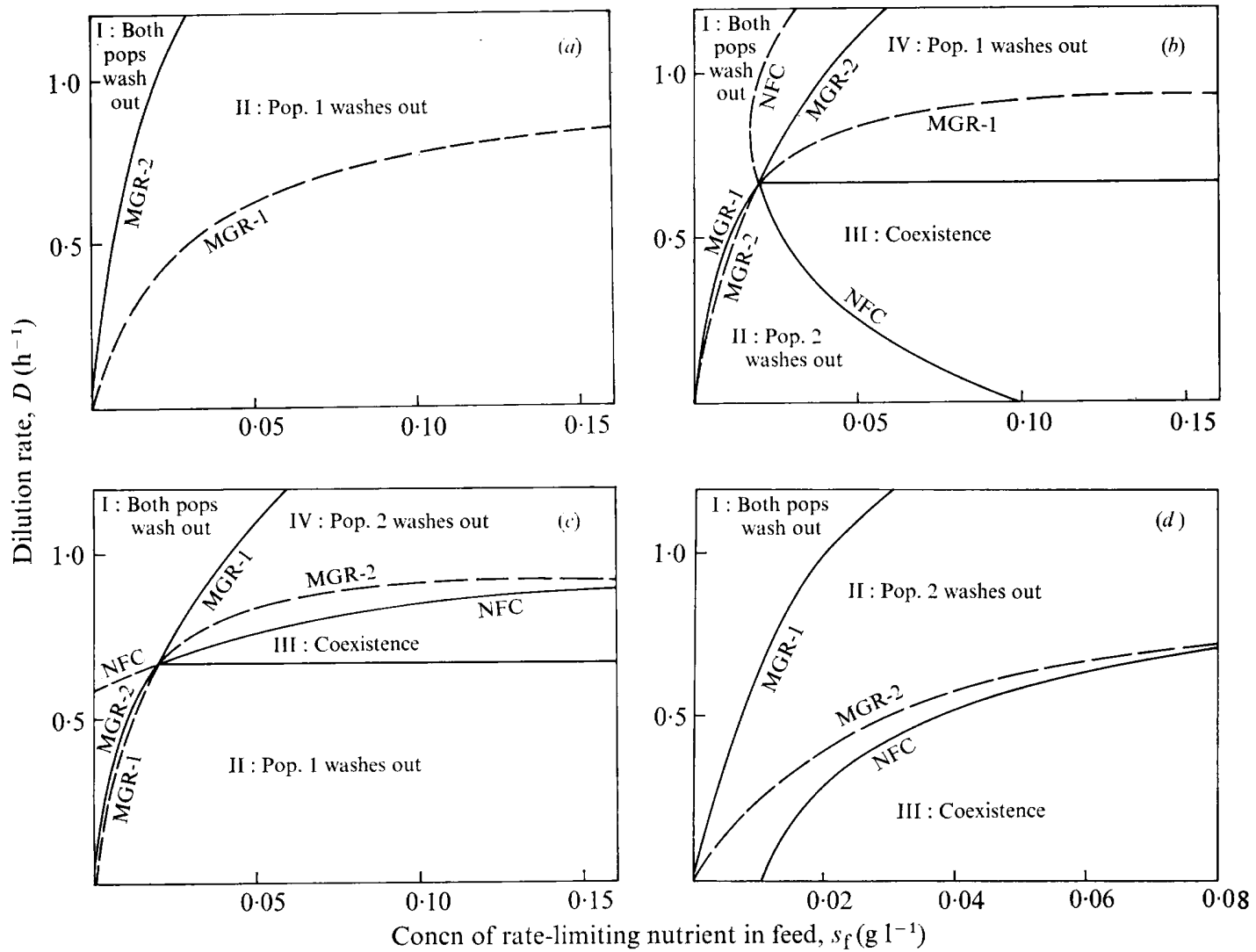

Fig. 3. Operating diagrams for Case II: population 1 produces a specific autoinhibitor. The values of the model parameters used in constructing these diagrams are as follows:

$\begin{array}{lcccccc} & \begin{array}{c}\mu_{\mathrm{m} 1} \\ \left(\mathrm{~h}^{-1}\right)\end{array} & \begin{array}{c}\mu_{\mathrm{m} 2} \\ \left(\mathrm{~h}^{-1}\right)\end{array} & \begin{array}{c}K_{1} \\ \left(\mathrm{~g} \mathrm{l}^{-1}\right)\end{array} & \begin{array}{c}K_{2} \\ \left(\mathrm{~g} \mathrm{l}^{-1}\right)\end{array} & \begin{array}{c}K_{1}^{\prime} \\ \left(\mathrm{g} \mathrm{l}^{-1}\right)\end{array} & \alpha_{1} Y_{1} \\ (a) & 1 \cdot 0 & 2 \cdot 0 & 0.03 & 0 \cdot 02 & - & - \\ (b) & 1 \cdot 0 & 2 \cdot 0 & 0.01 & 0.04 & 0 \cdot 0100 & 0 \cdot 1 \\ (c) & 2 \cdot 0 & 1 \cdot 0 & 0.04 & 0.01 & 0 \cdot 0005 & 0 \cdot 1 \\ (d) & 2 \cdot 0 & 1 \cdot 0 & 0.02 & 0.03 & 0 \cdot 0005 & 0 \cdot 1\end{array}$

The remaining model parameters are irrelevant to the construction of the diagrams.

Case III. The populations produce specific autoinhibitors

Here, each population is considered to produce an autoinhibitor that acts only on itself. If we denote the concentration in the culture of the inhibitor produced by the jth population as $i_{\mathrm{j}}$, the equations describing this situation are

$$
\begin{aligned}
& \frac{\mathrm{d} x_{\mathrm{j}}}{\mathrm{d} t}=-D x_{\mathrm{j}}+\mu_{\mathrm{j}} x_{\mathrm{j}}, \quad \mathrm{j}=1,2 \\
& \frac{\mathrm{d} i_{\mathrm{j}}}{\mathrm{d} t}=-D i_{\mathrm{j}}+\alpha_{\mathrm{j}} \mu_{\mathrm{j}} x_{\mathrm{j}} \\
& \frac{\mathrm{d} s}{\mathrm{~d} t}=D\left(s_{\mathrm{f}}-s\right)-\sum_{\mathrm{j}=1}^{2} \frac{1}{Y_{\mathrm{j}}} \mu_{\mathrm{j}} x_{\mathrm{j}}
\end{aligned}
$$

where the specific growth rates are given by

$$
\mu_{\mathrm{j}}=\frac{\mu_{\mathrm{mj}} s}{\left(K_{\mathrm{j}}+s\right)\left[1+i_{\mathrm{j}} / K_{\mathrm{j}}^{\prime}\right]}
$$

and the other quantities are as defined previously. 


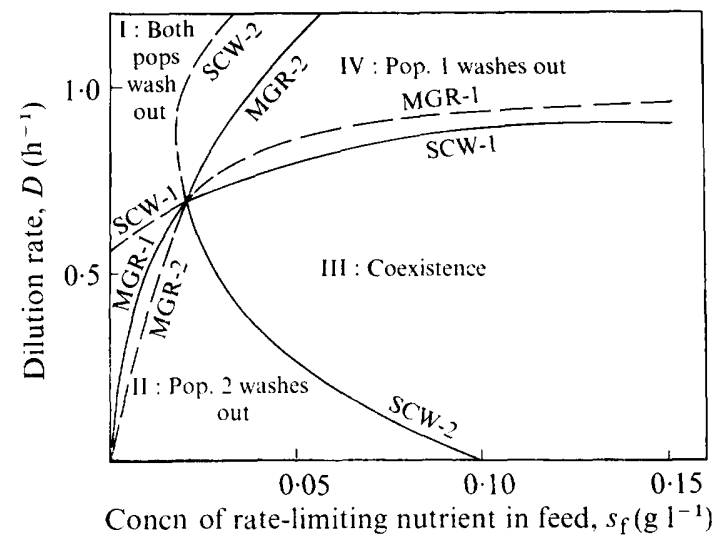

Fig. 4. Operating diagram for Case III: both populations produce specific autoinhibitors. The values of the model parameters used in constructing this diagram are as follows:

$\begin{array}{cccccc}\begin{array}{c}\mu_{\mathrm{m} 1} \\ \left(\mathrm{~h}^{-1}\right)\end{array} & \begin{array}{c}\mu_{\mathrm{m} 2} \\ \left(\mathrm{~h}^{-1}\right)\end{array} & \begin{array}{c}K_{1} \\ \left(\mathrm{~g} \mathrm{l}^{-1}\right)\end{array} & \begin{array}{c}K_{2} \\ \left(\mathrm{~g} \mathrm{l}^{-1}\right)\end{array} & \begin{array}{c}K_{1}^{\prime} / \alpha_{1} Y_{1} \\ \left(\mathrm{~g} \mathrm{l}^{-1}\right)\end{array} & \begin{array}{c}K_{2}^{\prime} / \alpha_{2} Y_{2} \\ \left(\mathrm{~g} \mathrm{l}^{-1}\right)\end{array} \\ 1.0 & 2 \cdot 0 & 0.01 & 0.04 & 0.1 & 0.03\end{array}$

Figure 4 shows an operating diagram constructed from these equations. The MGR curves of the two populations having the given parameters cross (at $D=0.667 \mathrm{~h}^{-1}$ and $s_{\mathrm{f}}=0.02 \mathrm{~g}^{-1}$ ) and the operating diagram would be that of Fig. 2 were it not for the actions of the autoinhibitors. In Fig. 4, the line of Fig. 2 along which coexistence is stable is expanded into region III in which coexistence is stable. The range of operating conditions in which coexistence is stable includes dilution rates both greater than and less than that at which the MGR curves cross. This contrasts with the situations shown in Figs $3(b)$ and 3(c) where the ranges of operating conditions in which coexistence is stable have, as one of their boundaries, the horizontal line that passes through the crossing point of the MGR curves. In effect, the actions of the specific autoinhibitors combine the operating diagrams of Figs $3(b)$ and $3(c)$ to produce that of Fig. 4. Evidently, if the populations were sufficiently affected by their autoinhibitors (i.e. if $K_{1}^{\prime}$ and $K_{2}^{\prime}$ were sufficiently small), coexistence would be possible under virtually all operating conditions that would allow pure cultures of both organisms to establish themselves. Thus, the production of specific autoinhibitors has a powerful stabilizing effect on the coexistence of competitors.

\section{Case IV. Inhibitors do not act on the populations that produce them}

In the previous three cases, a substance produced by a population had an inhibitory effect on that population. We now assume that each population produces a substance that has an inhibitory effect on the competing population, but not on itself. Thus, the two populations interact in two ways: they compete for the rate limiting nutrient, and they interfere with each other's growth through the inhibition (interference-type competition; Odum, 1971).

The equations for this situation are, once again, equations $(2 a),(2 b)$ and $(2 c)$, but equation $(2 d)$ must be replaced by

where $\mathrm{j}, \mathrm{k}=1,2$ and $\mathrm{j} \neq \mathrm{k}$.

$$
\mu_{\mathrm{j}}=\frac{\mu_{\mathrm{mj}} s}{\left(K_{\mathrm{j}}+s\right)\left[1+i_{\mathrm{k}} / K_{\mathrm{j}}^{\prime}\right]}
$$

Coexistence steady state solutions of equations $(2 a),(2 b),(2 c)$ and (3) exist for a range of operating conditions in the first quadrant of the $D-s_{f}$ plane, but these steady states are always unstable. Hence, the competitors cannot coexist in this case, and the only steady states of concern are those in which both populations wash out or in which one population excludes the other.

Figure 5 shows operating diagrams for two sets of model parameters for this case. The 


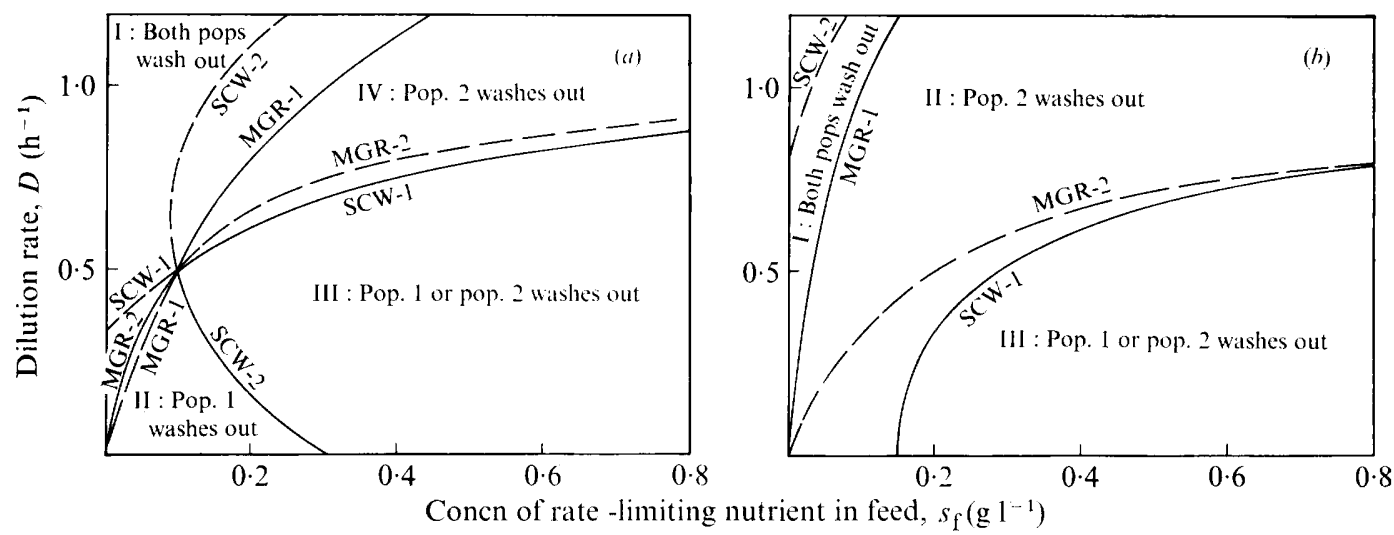

Fig. 5. Operating diagrams for Case IV: each population produces an inhibitor which acts on its competitor but not on itself. The values of the model parameters used in constructing these diagrams are as follows:

(a)

(b)

$$
\begin{gathered}
\mu_{\mathrm{m} 1} \\
\left(\mathrm{~h}^{-1}\right)
\end{gathered}
$$$$
2 \cdot 0
$$

$2 \cdot 0$

$$
\begin{gathered}
\mu_{\mathrm{m} 2} \\
\left(\mathrm{~h}^{-1}\right) \\
1 \cdot 0
\end{gathered}
$$

$1 \cdot 0$

$K_{1}$
$\left(\mathrm{~g} \mathrm{l}^{-1}\right)$
0.3

$0 \cdot 1$

$K_{2}$
$\left(\mathrm{~g} \mathrm{l}^{-1}\right)$
$0 \cdot 1$

$0 \cdot 2$

boundary curves in these diagrams are formed from the MGR curves and from the curves that express conditions for the stability of the steady states where population 1 is washed out (SCW-1) or population 2 is washed out (SCW-2); see Appendix for further discussion of these curves.

In both parts of Fig. 5 there is a range of operating conditions, region III, where two steady states are stable with respect to small perturbations. These are the steady states where population 1 excludes population 2 and where population 2 excludes population 1 . The results of competition under these operating conditions would depend on the initial conditions of the culture. Thus, even though inhibition effects cannot in this case allow the competitors to coexist, they are nevertheless sufficient to give a population a chance for survival under conditions where it would have no such chance if no inhibitors were present. For instance, in Fig. 5(a), population 1 would be excluded at all dilution rates less than $0.5 \mathrm{~h}^{-1}$, and population 2 would be excluded at all dilution rates greater than $0.5 \mathrm{~h}^{-1}$, if there were no inhibition effects. Similarly, in Fig. 5(b), population 2 could not exclude population 1 at any dilution rate if there were no inhibition effects.

The diagrams shown in Fig. 5 do not exhaust the possibilities for constructing qualitatively different operating diagrams for Case IV. Rather than trying to construct all such diagrams, we have considered only one specialized version of this case, Case V.

\section{Case V. Amensalism plus competition}

This special version of Case IV arises when one of the populations is not affected by the inhibitor produced by the other. The interactions between the populations would be a combination of amensalism and competition. Analysis can proceed either from equations $(2 a),(2 b),(2 c)$ and (3) by letting, say, $K_{1}^{\prime}$ approach infinity, or from equations (1) by letting $K_{1}^{\prime}$ become infinite and $\alpha_{2}$ become zero, and then setting $i$ equal to $i_{1}$. Four qualitatively different operating diagrams arise (Fig. 6); none of these shows that a stable coexistence steady state is possible. However, antagonism of population 1 toward population 2 gives the former a chance to exclude the latter under conditions where the former would have no chance to survive were it not for the antagonism. This is the case when operating conditions lie in region III of Figs $6(a), 6(b)$ and $6(c)$.

Thus, the effect of antagonism is to increase the number of possible outcomes of competition but not to allow coexistence. 

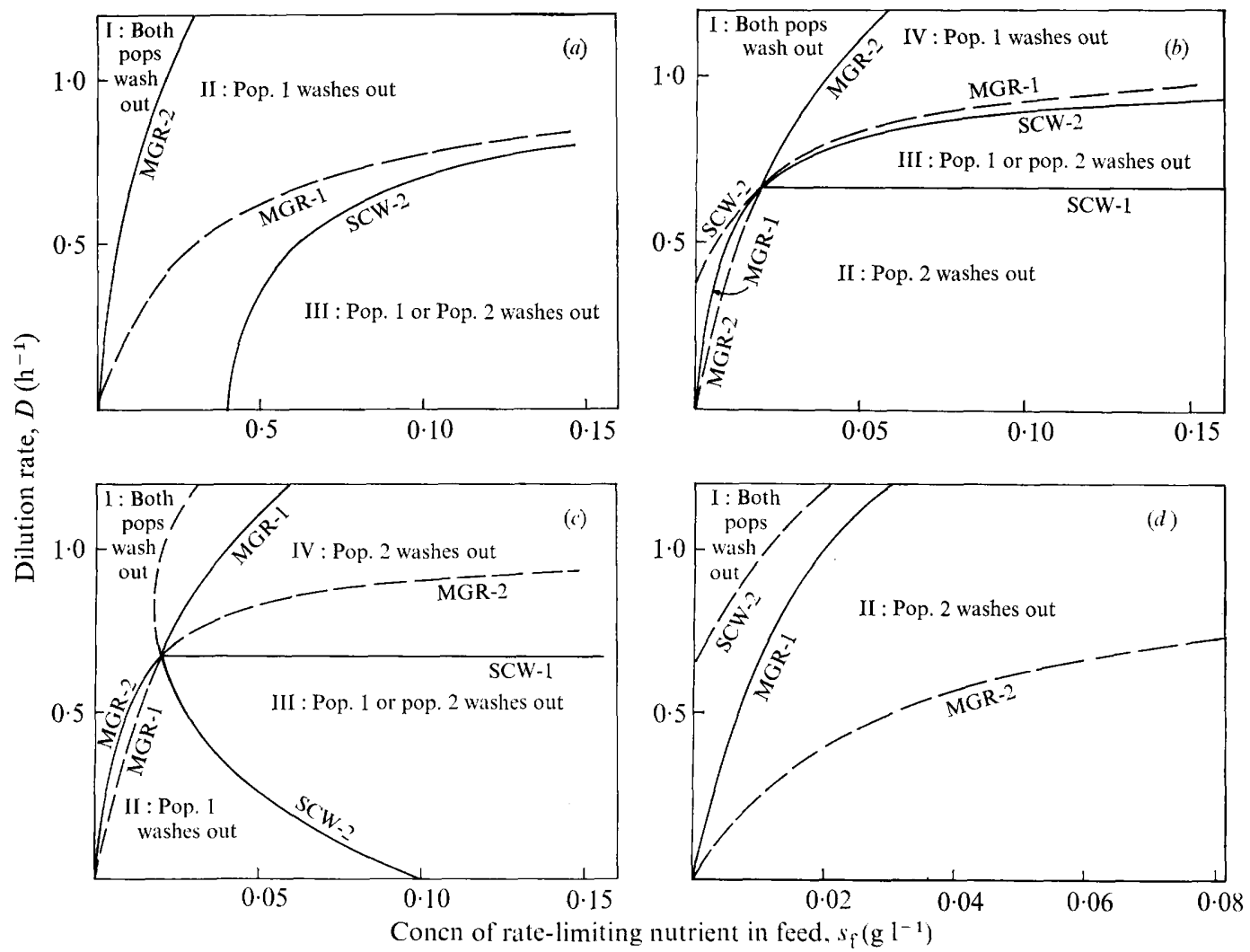

Fig. 6. Operating diagrams for Case V: population 1 produces an inhibitor that acts on population 2 but not on itself. The values of the model parameters used in constructing these diagrams are as follows:

$\begin{array}{lccccc} & \begin{array}{c}\mu_{\mathrm{m} 1} \\ \left(\mathrm{~h}^{-1}\right)\end{array} & \begin{array}{c}\mu_{\mathrm{m} 2} \\ \left(\mathrm{~h}^{-1}\right)\end{array} & \begin{array}{c}K_{1} \\ \left(\mathrm{~g} \mathrm{l}^{-1}\right)\end{array} & \begin{array}{c}K_{2} \\ \left(\mathrm{~g} \mathrm{l}^{-1}\right)\end{array} & \begin{array}{c}K_{2}^{\prime} / \alpha_{1} Y_{1} \\ \left(\mathrm{~g} \mathrm{l}^{-1}\right)\end{array} \\ (a) & 1.0 & 2.0 & 0.03 & 0.02 & 0.04 \\ (b) & 1.0 & 2.0 & 0.01 & 0.04 & 0.02 \\ (c) & 2.0 & 1.0 & 0.04 & 0.01 & 0.10 \\ (d) & 2.0 & 1.0 & 0.02 & 0.03 & 0.01\end{array}$

The remaining model parameters are irrelevant to the construction of the diagrams.

\section{DISCUSSION}

The models presented show that production of a secondary substance having an autoinhibitory effect will allow two populations that compete for a primary rate limiting nutrient to coexist in a stable steady state even when operating variables are not constrained to certain fixed values. Coexistence occurs because autoinhibition makes a population's growth rate partially self-regulated, and thus competitors can have the same specific growth rate over a range of concentrations of the rate limiting nutrient.

Production of a secondary substance that has inhibitory effects on other populations (antagonistic effects), but not on the population that produces it, will not allow competitors to coexist. Production of such substances will, however, increase the number of possible outcomes of competition and confer a chance for survival on a population that would have no such chance were it not for its production of an antagonistic substance.

Can production of secondary substances having autoinhibitory effects play a role in maintaining the species diversity of an ecosystem that is spatially homogeneous and has inputs and external circumstances that are time invariant? The pattern of Case I (production 


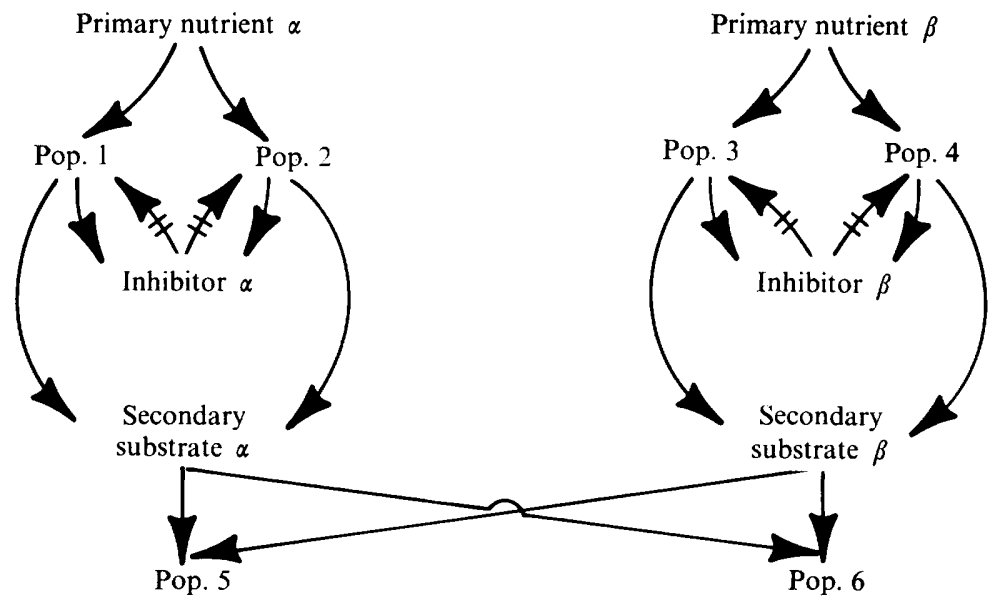

Fig. 7. Network of non-predatory interactions that should allow an input containing only two nutrients in rate limiting concentrations to support coexistence of six populations in a stable steady state.

of a common autoinhibitor by a set of populations that compete for a single primary rate limiting nutrient) will not allow coexistence of more than two competing populations, since there are only two environmental substances affecting the growth rates. The pattern of Cases II and III (production of specific autoinhibitors by a set of populations that compete for a single primary rate limiting nutrient) seems to have much greater potential for maintaining species diversity. In the two population situations of Cases II and III, the results suggest (though do not prove) that the number of competing populations that can coexist in a stable steady state is equal to one plus the number of specific autoinhibitors formed. If, therefore, there is no limit to the number of populations that can produce specific autoinhibitors, there would be no limit to the potential of this pattern for maintaining species diversity of ecosystems.

Production of secondary substances that have autoinhibitory effects can probably combine with production of secondary substances that have stimulatory effects to aid in maintaining species diversity of ecosystems. For example, Fig. 7 shows a hypothetical network of non-predatory interactions which could allow an input containing only two nutrients in rate limiting concentrations to support coexistence of six populations in a stable steady state, even though the system is spatially homogeneous and the inputs are time invariant. Even greater diversity would seem to be possible if additional secondary substances were formed.

The above considerations prove nothing about any specific microbial ecosystem. They do suggest, however, that production of secondary substances in general, and of autoinhibitory secondary substances in particular, can play a significant role in maintaining the species diversity of such systems.

\section{APPENDIX}

\section{Construction of operating diagrams}

Case I. Equations (1) admit of three different kinds of steady states: washout of both populations; one population is excluded but the other one establishes itself; and the populations coexist. There are two steady states of the second kind and, depending on model parameters, zero, one or two steady states of the third kind.

Determination of the conditions under which these various steady states are real (all concentrations nonnegative) and stable can be reduced to a series of problems concerned with the properties of a pair of curves and a pair of lines in the $i-s$ plane. The curves are rate curves (denoted R-1 and R-2 on Fig. 8) which are simply the graphs of the steady state conditions $D=\mu_{\mathrm{j}}(i, s)$, where $\mathrm{j}=1,2$. The lines are stoicheiometric 


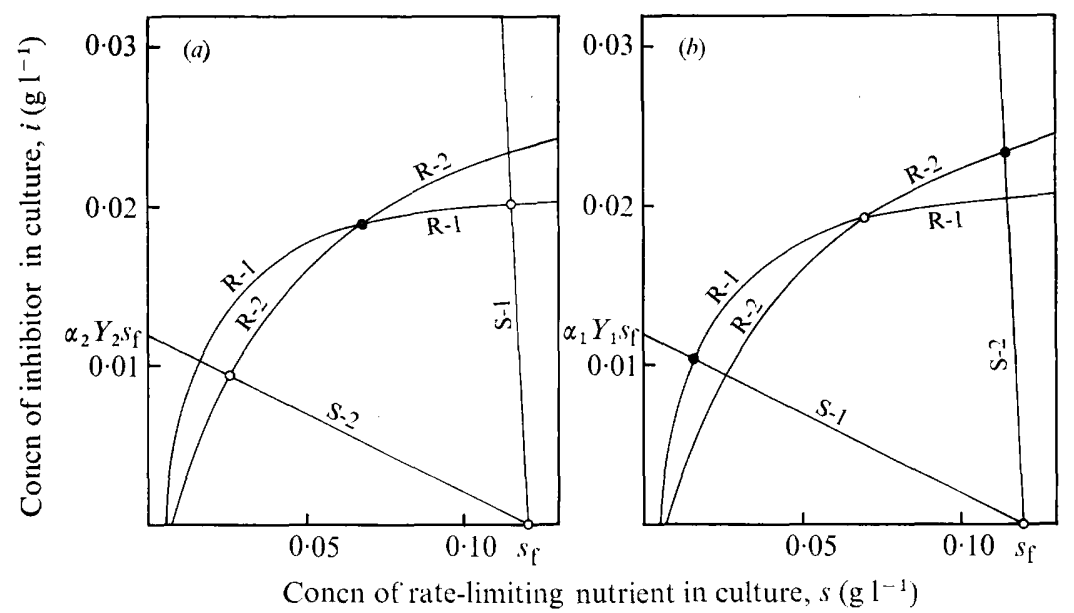

Fig. 8. Graphical procedure used to determine the nature of the steady states of equations (1) for a fixed set of model parameters and fixed operating conditions. The model parameters used for $(a)$ and $(b)$ are the same as those used to construct the operating diagrams shown in Figs $1(a)$ and $1(b)$, respectively. The operating conditions are $D=0.3 \mathrm{~h}^{-1}$ and $s_{\mathrm{t}}=0.12 \mathrm{~g} \mathrm{l}^{-1}$. Stable steady states are denoted by filled circles and unstable steady states are denoted by open circles. Note that in both $(a)$ and $(b)$, four steady states are meaningful but only one is stable.

lines (denoted by S-1 and S-2 on Fig. 8) which are the graphs of the steady state relations between $i$ and $s$ which must be satisfied for steady states of the second kind (exclusion of one population). The equation of $\mathrm{S}-\mathrm{j}$ is $i=\alpha_{\mathrm{j}} Y_{\mathrm{j}}\left(s_{\mathrm{i}}-s\right)$.

It can be seen from equations $(1 a),(1 b)$ and $(1 c)$ that the quantities $\left(i-\alpha_{1} x_{1}-\alpha_{2} x_{2}\right)$ and $\left[s_{f}-s-\left(x_{1} / Y_{1}\right)-\right.$ $\left.\left(x_{2} / Y_{2}\right)\right]$ both approach zero as $t$ approaches infinity, regardless of the initial values of these quantities, and regardless of the operating conditions or the model parameters. Hence, there are really only two dependent variables in equations (1), and the characteristic equation of the stability matrix for these equations is a quadratic, rather than a quartic. Moreover, the roots of this quadratic are always real for a real steady state, so that focal type behaviour (oscillations) is not predicted by the model.

The steady state of the first kind, washout of both populations, is asymptotically stable with respect to small perturbations if the dilution rate exceeds the MGR of both populations at the value of $s_{\mathrm{f}}$ used; otherwise it is unstable.

The values of $i$ and $s$ for a steady state of the second kind, say the one where population 2 is excluded, are the values of $i$ and $s$ at the intersection of the R-1 curve and the S-1 line. No more than one such intersection can occur in the first quadrant of the $i-s$ plane. If an intersection does occur in the first quadrant, it will be such that $0<s<s_{\mathrm{f}}$ and, since $x_{1}=Y_{1}\left(s_{\mathrm{f}}-s\right)$ for this steady state, such an intersection must correspond to a real steady state. Linearized stability analysis shows that this steady state will be asymptotically stable with respect to small perturbations if the ordinate of the R-1 curve lies above the ordinate of the R-2 curve at the value of $s$ corresponding to the intersection; the steady state is unstable otherwise. Exactly similar considerations apply to the steady state of the second kind where population 1 is excluded. Intersections for both steady states of this kind are shown in Fig. 8; in Fig. 8(a), these steady states are unstable, but in Fig. $8(b)$ they are stable.

The values of $i$ and $s$ for a steady state of the third kind, coexistence of the two populations, are the values of these quantities at an intersection of the R-1 curve with the R-2 curve. These curves may intersect once, twice, or not at all, in the first quadrant of the $i-s$ plane, depending on the values of the model parameters; in Fig. 8, a single intersection is shown. For such an intersection to be that of a real steady state, it must (i) lie in the first quadrant of the $i-s$ plane so that $i$ and $s$ will be positive, and (ii) lie between the S-1 line and the S-2 line so that $x_{1}$ and $x_{2}$ will be positive.

A real coexistence steady state is asymptotically stable with respect to small perturbations if

$$
\left(\alpha_{1} Y_{1}-\alpha_{2} Y_{2}\right)\left[K_{2}\left(K_{1}+s\right)\left(K_{2}^{\prime}+i\right)-K_{1}\left(K_{2}+s\right)\left(K_{1}^{\prime}+i\right)\right]>0
$$

or, in view of equation $(1 d)$, if

$$
\left(\alpha_{1} Y_{1}-\alpha_{2} Y_{2}\right)\left[\left(\frac{\partial i}{\partial s}\right)_{\mu_{2}}-\left(\frac{\partial i}{\partial s}\right)_{\mu_{1}}\right]>0
$$


The derivative $(\partial i / \partial s)_{\mu}$ is, of course, the slope of the R-j curve on Fig. 8. In that figure, $(\partial i / \partial s)_{\mu_{2}}>(\partial i / \partial s)_{\mu_{1}}$ at the intersection of the R-1 and R-2 curves, so that coexistence is stable in Fig. 8(a) where $\left(\alpha_{1} Y_{1}-\alpha_{2} Y_{2}\right)>0$ but it is unstable in Fig. $8(b)$ where $\left(\alpha_{1} Y_{1}-\alpha_{2} Y_{2}\right)<0$.

It is important to note that the R-1 and R-2 curves are independent of $s_{\mathrm{f}}$ whereas the S-1 and S-2 lines are independent of $D$.

Operating conditions under which a coexistence steady state will be stable and real, for a given set of model parameters, can be easily determined using the facts outlined above. One first constructs plots of R-1 and R-2 curves for a series of values of $D$, and from the changing location of the intersection of these curves one determines how the steady state values of $i$ and $s$ change with $D$. The range of $s_{t}$ values that will make any first quadrant intersection of the R-1 and R-2 curves determine a real steady state is found by drawing straight lines of slope $-\alpha_{1} Y_{1}$ and $-\alpha_{2} Y_{2}$ through the intersection and extending them to the points where they intercept the line $i=0$. All values of $s_{\mathrm{f}}$ lying between these intercepts will produce real coexistence steady states. This range of $s_{f}$ values must be determined for each value of $D$ used. The stability of a real coexistence steady state is determined by the condition on the slopes of the R-1 and R-2 curves given above. This procedure was used to construct the operating diagrams shown in Fig. 1.

Case II. This is a special case of Case I and the graphical methods described above may be used to construct operating diagrams. In the situation where the autoinhibitor is specific to population 1, the R-2 curve in the $i-s$ plane is the vertical line $s=D K_{2} /\left(\mu_{\mathrm{m} 2}-D\right)$ and the S-2 line is the horizontal line $i=0$. The condition that the coexistence steady state be real may be expressed analytically in this case; it is that

$$
s_{\mathrm{f}}>\frac{D K_{2}}{\mu_{\mathrm{m} 2}-D}+\frac{K_{1}^{\prime}}{\alpha_{1} Y_{1}}\left[\frac{\left(K_{2} \mu_{\mathrm{m} 1}-K_{1} \mu_{\mathrm{m} 2}\right)-\left(K_{2}-K_{1}\right) D}{K_{1} \mu_{\mathrm{m} 2}+\left(K_{2}-K_{1}\right) D}\right]
$$

The loci of operating conditions along which this inequality is just satisfied are labelled NFC (for Nutrient Feed Condition) in Fig. 3.

Case III. It can be seen from equations (2) that the quantities $\left(i_{1}-\alpha_{1} x_{1}\right),\left(i_{2}-\alpha_{2} x_{2}\right)$ and $\left[s_{f}-s-\left(x_{1} / Y_{1}\right)-\right.$ $\left(x_{2} / Y_{2}\right)$ ] all approach zero as $t$ approaches infinity, regardless of the initial values of these quantities, and regardless of the operating conditions or the model parameters. Hence, there are really only two dependent variables in equations (2), and the characteristic equation of the stability matrix for these equations is a quadratic one, rather than one of fifth degree. Moreover, the roots of this quadratic are always real for a real steady state, so that oscillations are not predicted by the model.

Equations (2) admit four steady state solutions: washout of both populations; washout of population 2; washout of population 1; and coexistence. The nutrient concentrations for these steady states are given by

$$
\begin{gathered}
s_{\mathrm{f}}-s=0 \quad \text { when both populations wash out, } \\
s_{\mathrm{f}}-s=\frac{K_{1}^{\prime}}{\alpha_{1} Y_{1}}\left[\frac{\left(\mu_{\mathrm{m} 1} / D\right) s}{K_{1}+s}-1\right] \text { when population } 2 \text { washes out, } \\
s_{\mathrm{f}}-s=\frac{K_{2}^{\prime}}{\alpha_{2} Y_{2}}\left[\frac{\left(\mu_{\mathrm{m} 2} / D\right) s}{K_{2}+s}-1\right] \text { when population } 1 \text { washes out }
\end{gathered}
$$

and $\quad s_{\mathrm{f}}-s=\frac{K_{1}^{\prime}}{\alpha_{1} Y_{1}}\left[\frac{\left(\mu_{\mathrm{m} 1} / D\right) s}{K_{1}+s}-1\right]+\frac{K_{2}^{\prime}}{\alpha_{2} Y_{2}}\left[\frac{\left(\mu_{\mathrm{m} 2} / D\right) s}{K_{2}+s}-1\right]$ when the populations coexist.

The steady state where both populations wash out will be stable if $D>\max \left[\mu_{\mathrm{m} 1} s_{\mathrm{f}} /\left(K_{1}+s_{\mathrm{f}}\right), \mu_{\mathrm{m} 2} s_{\mathrm{f}} /\right.$ $\left(K_{2}+s_{t}\right)$ ], that is, if $D$ exceeds the MGR of both populations. The steady state where population 2 washes out will be real if $0<s<s_{\mathrm{f}}$ and stable if $D>\mu_{\mathrm{m}_{2}} s /\left(K_{2}+s\right)$, where $s$ is, of course, the function of $D$ and $s_{1}$ given by the second of the steady state relations above. Elimination of $s$ from the steady state relation and the stability condition allows one to express the stability condition in terms of $D$ and $s_{f}$; the locus of operating conditions where this condition is just satisfied is the curve marked SCW-2 (Stability Condition for Washout of population 2) in Fig. 4. The analogous curve SCW-1 is constructed in the same way.

The coexistence steady state will be real if $0<s<s_{\mathrm{f}}$ and $D<\min \left[\mu_{\mathrm{m} 1} s /\left(K_{1}+s\right), \mu_{\mathrm{m} 2} s /\left(K_{2}+s\right)\right]$, where $s$ is the function of $D$ and $s_{t}$ given by the last of the steady state relations above. The coexistence steady state is stable if it is real. Expression of the conditions for the coexistence steady state to be real (which are also the stability conditions) in terms of the operating variables $D$ and $s_{f}$ leads to exactly the same relations as found for the stability conditions of the steady states where one population washes out, but with the senses of the inequalities reversed. Thus, the curves SCW-1 and SCW-2 of Fig. 4 are the boundary curves for the range of operating conditions where coexistence is stable.

Case IV. From equations $(2 a),(2 b)$ and $(2 c)$, it can be seen that the quantities $\left(i_{1}-\alpha_{1} x_{1}\right),\left(i_{2}-\alpha_{2} x_{2}\right)$ and $\left[s_{t}-s-\left(x_{1} / Y_{1}\right)-\left(x_{2} / Y_{2}\right)\right]$ all approach zero as $t$ approaches infinity, regardless of the initial values of these 
quantities, and regardless of the operating conditions or the model parameters. Hence, there are really only two independent variables in equations $(2 a),(2 b),(2 c)$ and (3), and so the characteristic equation of the stability matrix for these equations is a quadratic one, rather than one of fifth degree. Moreover, the roots of this quadratic are always real for a real steady state.

The coexistence steady state is always unstable when it is real. The conditions that are necessary and sufficient for the steady state where population 1 excludes population 2 to be real and stable with respect to small perturbations are

$$
\frac{\mu_{\mathrm{m} 1} s_{t}}{K_{1}+s_{t}}>D>\frac{\mu_{\mathrm{m} 2} D K_{1} /\left(\mu_{\mathrm{m} 1}-D\right)}{\left[K_{2}+D K_{1} /\left(\mu_{\mathrm{m} 1}-D\right)\right]\left\{1+\left(\alpha_{1} Y_{1} / K_{2}^{\prime}\right)\left[s_{t}-D K_{1} /\left(\mu_{\mathrm{m} 1}-D\right)\right]\right\}}
$$

The conditions that are necessary and sufficient for the steady state where population 2 excludes population 1 to be real and stable with respect to small perturbations may be found from the foregoing conditions simply by interchanging the subscripts 1 and 2 . The loci of operating conditions where the left-hand inequality above is just satisfied is the MGR-1 curve. The loci of operating conditions where the right-hand inequality above is just satisfied are the SCW-2 (Stability Condition for Washout of population 2) curves on Fig. 5.

Case V. This is a special case of Case IV, and the above discussion of that case will also apply here.

\section{REFERENCES}

Arba, S. \& ShodA, M. (1969). Reassessment of the product inhibition in alcohol fermentation. Journal of Fermentation Technology 47, 790-794.

ARIS, R. \& Humphrey, A. E. (1977). Dynamics of a chemostat in which two organisms compete for a common substrate. Biotechnology and Bioengineering 19, 1375-1386.

HARDeR, W. \& VeldKamp, H. (1971). Competition of marine psychrophilic bacteria at low temperatures. Antonie van Leeuwenhoek 37, 51-63.

HARdiN, G. (1960). The competitive exclusion principle. Science 131, 1292-1297.

Hutchinson, G. E. (1961). The paradox of the plankton. American Naturalist 95, 137-145.

JANNASCH, H. W. (1967). Enrichments of aquatic bacteria in continuous culture. Archiv für Mikrobiologie 59, 165-173.

JANNASCH, H. W. (1968). Competitive eliminations of Enterobacteriaceae from seawater. Applied Microbiology 16, 1616-1618.

Jost, J. L., Drake, J. F., Fredrickson, A. G. \& TsuchiYA, H. M. (1973). Interactions of Tetrahymena pyriformis, Escherichia coli, Azotobacter vinelandii, and glucose in a minimal medium. Journal of Bacteriology 113, 834-840.

Lee, I. H., Fredrickson, A. G. \& Tsuchiya, H. M. (1976). Dynamics of mixed cultures of Lactobacillus plantarum and Propionibacterium shermanii. Biotechnology and Bioengineering 18, 513526.

MeERs, J. L. (1971). Effect of dilution rate on the outcome of chemostat mixed culture experiments. Journal of General Microbiology 67, 359-361.

Meers, J. L. \& Tempest, D. W. (1968). The influence of extracellular products on the behaviour of mixed microbial populations in magnesiumlimited chemostat cultures. Journal of General Microbiology 52, 309-317.
Megee, R. D., III, Drake, J. F., Fredrickson, A. G. \& TsuchiYA, H. M. (1972). Studies in intermicrobial symbiosis. Saccharomyces cerevisiae and Lactobacillus casei. Canadian Journal of Microbiology 18, 1733-1742.

Meyer, J.S., Tsuchiya, H. M., \& Fredrickson, A. G. (1975). Dynamics of mixed populations having complementary metabolism. Biotechnology and Bioengineering 17, 1065-1081.

ODum, E. P. (1971). Fundamentals of Ecology, 3rd edn, p. 211. Philadelphia: W. B. Saunders.

Powell, E. O. (1958). Criteria for the growth of contaminants and mutants in continuous culture. Journal of General Microbiology 18, 259-268.

SCHUELKE, L. M. (1976). Stability analysis of various chemical engineering systems by a method of decomposition. Unpublished M.S. Thesis, University of Minnesota.

TAYlor, P. A. \& Williams, P. J. LeB. (1975). Theoretical studies on the coexistence of competing species under continuous-flow conditions. Canadian Journal of Microbiology 21, 90-98.

Tempest, D. W., Dicks, J. W. \& MeERs, J. L. (1967). Magnesium-limited growth of Bacillus subtilis, in pure and mixed cultures, in a chemostat. Journal of General Microbiology 49, 139-147.

VAN GEMERDEN, H. (1974). Coexistence of organisms competing for the same substrate: an example among the purple sulfur bacteria. Microbial Ecology 1, 104-119.

Wilkinson, T. G., Topiwala, H. H. \& Hamer, G. (1974). Interactions in a mixed bacterial population growing on methane in continuous culture. Biotechnology and Bioengineering 16, 41-59.

Williams, F. M. (1971). Dynamics of microbial populations. In Systems Analysis and Simulation in Ecology, vol. I, pp. 198-267. Edited by B. C. Patten. New York: Academic Press. 